\title{
Mesh Erosion and Sigmoido-vaginal Fistula
}

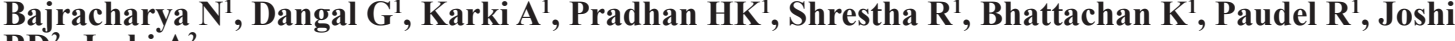 \\ $\mathrm{BD}^{2}$, Joshi $\mathrm{A}^{2}$ \\ ${ }^{1}$ Department of Obstetrics and Gynecology, Kathmandu Model Hospital, Kathmandu, Nepal, ${ }^{2}$ Department of General Sur- \\ gery, Kathmandu Model Hospital, Kathmandu, Nepal.
}

Received: 19-May-2017; Accepted: 30-May-2017

\begin{abstract}
Abdominal sacro-colpopexy (ASC) is a commonly performed procedure for the surgical treatment of pelvic organ prolapse (POP). Surgeons favor this technique because of its well-established success rates ranging from $78-100 \%$ and durability, which is attributed to reinforcement of native tissues with a graft. Despite recognition of the risk of erosion, synthetic grafts have been preferred over autologous grafts for abdominal sacro-colpopexy because they are durable, avoid the morbidity and operative time of harvesting fascia, are readily available and are relatively inexpensive. Ideally, these benefits should outweigh the possibility of erosion, which is a unique complication of using a graft.
\end{abstract}

We present here a unique case of mesh erosion into bowel after sacro-colpopexy who presented with sigmoido-vaginal fistula.

Keywords: mesh erosion; sacrocolopopexy; sigmoido-vaginal fistula; synthetic grafts.

DOI: http://dx.doi.org/10.3126/njog.v12i1.18985

\section{INTRODUCTION}

Pelvic organ prolapse (POP) procedures are relatively common. One of the most widely accepted procedures for apical prolapse is the abdominal sacro-colpopexy (ASC). The ASC uses synthetic or biologic graft material to suspend the vaginal vault to the anterior longitudinal ligament of the sacrum. It is associated with a long-term success rate of $84-99 \%$ and is considered the gold standard. ${ }^{1}$

One of the most troublesome complication of ASC is vaginal mesh erosion, with incidence of $3.4 \% .{ }^{2}$ The rate of vaginal mesh erosion has been shown to be affected adversely by patient's smoking status, the type of mesh used, and the surgical technique. ${ }^{3}$ The Pelvic Floor Disorders Network reported an increased risk of erosion in patients who have a concomitant hysterectomy ( $60 \%$ vs $24 \%$ ) based on Colpopexy And urinary Reduction Efforts (CARE) trial. ${ }^{4} \mathrm{We}$ present a rare case of mesh erosion into bowel after ASC.

\section{CASE}

A 55years lady came with complaint of passage of stool per vagina since onemonth associated with foul smelling discharge and itching for the past one year. She underwent abdominal hysterectomy 14

\section{CORRESPONDENCE}

Dr Nishma Bajracharya

Department of Obstetrics and Gynaecology

Kathmandu Model Hospital, Kathmandu, Nepal.

Phone: +977-9860157654

Email: nishu_bajra@hotmail.com years back for chronic cervicitis with prophylactic abdominal sacrocolpopexy with mesh placement. No history of any vaginal surgery, radiation therapy or trauma was given.

Per abdominal exam revealed soft and non-tender abdomen, per speculum and per vaginal examination revealed presence of stool in vagina with a one $\mathrm{cm}$ defect in vault area with mesh like structure in situ (Figure 1).Colonoscopy done showed stricture at sigmoid coon at $30 \mathrm{~cm}$ proximal to anal verge.

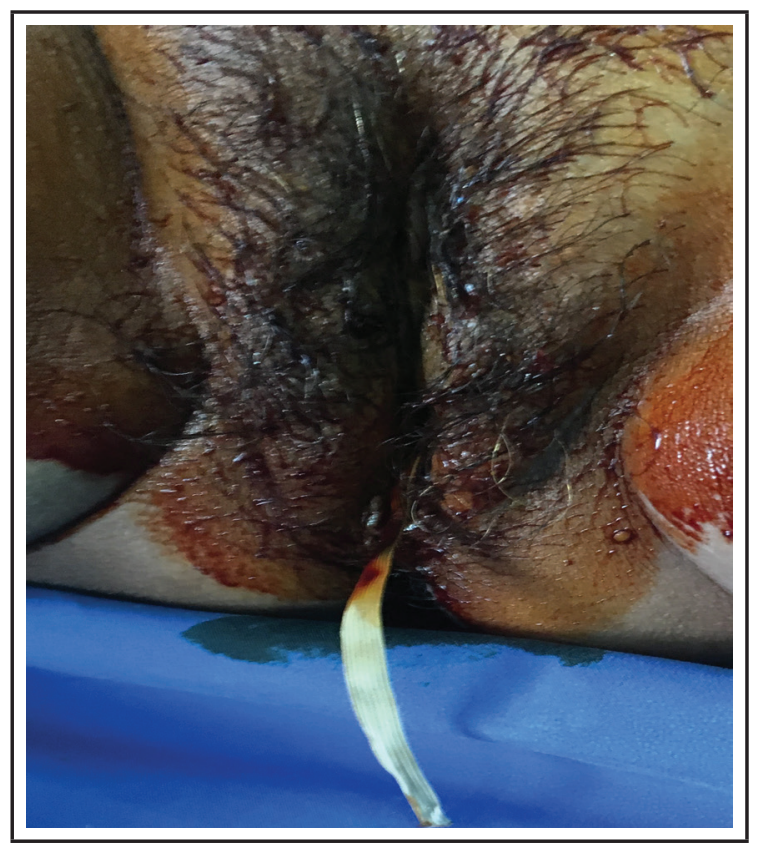

Figure 1. Mesh coming out of vagina through a defect in vault. 
She underwent Exploratory Laparatomy with adhesiolysis with take down of sigmoido vaginal fistula with loop colostomy. Intraoperatively, a fistulous tract was found between vagina and mid sigmoid colon with one $\mathrm{cm}$ defect, the defect in sigmoid colon was brought out as temporary colostomy and planned to reverse colostomy after three months. Patient was discharged with colostomy with diagnosis of sigmoido-vaginal fistula secondary to mesh erosion.

\section{COMMENTS}

The performance of ASC for the surgical treatment of post-hysterectomy vaginal vault prolapse or complete uterine prolapse is well established. Suspension of the prolapsed vaginal vault to the anterior surface of the sacrum with graft material (mesh) was first reported by Lane. ${ }^{5}$ Timmons and Add is on reported a mesh erosion rate of $3.3 \%$, Snyder and Krantz reported a similar erosion rate of $2.7 \%$ and Kohli et al recently reported a mesh erosion rate of $8.8 \%$ after abdominal sacral colpopexy with the use of synthetic mesh. ${ }^{6}$

Sacro-colpopexy mesh erosion is typically evident by exposure of the graft in the vagina. In such cases, granulation tissue and a sero-purulent or serosanginous discharge is usually present, which may be accompanied by pain or tenderness and dyspareunia. Sometimes, the sacrocolpopexy vaginal sutures may be the only visible foreign material. Mesh Erosion may result from an inflammatory reaction due to infection of the foreign body or, possibly, due to an immunological response to the graft or suture material. Alternatively, the mesh or sutures may be exposed without an obvious inflammatory reaction and can be relatively asymptomatic. ${ }^{6}$

If mesh erosion occurs into hollow viscera, fistula may be formed, for example, vesicovaginal fistula in cases of erosions in urinary bladder (Category 4, IUGA/ICS classification) and rectovaginal or sigmoido-vaginal fistula in cases of erosions in bowel (Category 5, IUGA/ICS classification). ${ }^{7}$ In cases of mesh erosion with concomitant hysterectomy, the site is nearly always at the vaginal cuff. This may be due to increased vaginal bacterial contamination of the mesh from an opened vagina during hysterectomy, or poor healing at the new cuff because of the devascularizing effects of mesh vaginal attachment sutures.

Such erosion has been managed successfully from a vaginal approach by removing the exposed mesh or sinus tract and undermining the edges of the vagina, which are then sutured in multiple layers. Laparotomy with complete removal of the mesh may be required in refractory cases or if the mesh becomes infected. ${ }^{8}$

\section{CONCLUSIONS}

Mesh-related complications are a current emerging problem, which confronts all of us in our daily practice. Studies have shown that early surgical treatment of these complications is advantageous.

\section{CONFLICT OF INTEREST}

None

\section{REFERENCES}

1. Nosti PA, Lowman JK, Zollinger TW, Hale DS, Woodman PJ Risk of mesh erosion after abdominal sacral colpoperineopexy with concomitant hysterectomy. AJOG. 2009;201(5):541. e1e4.

2. Nygaard IE, McCreery R, Brubaker L, Connolly A, Cundiff G, Weber AM, et al. Abdominal sacrocolpopexy: a comprehensive review. Obstet Gynecol. 2004;104(4):805-23.

3. Lowman JK, Woodman PJ, Nosti PA, Bump RC, Terry CL, Hale DS. Tobacco use is a risk factor for mesh erosion after abdominal sacral colpoperineopexy. AJOG. 2008;198(5):561. e1-. e4

4. Cundiff GW, Varner E, Visco AG, Zyczynski HM, Nager $\mathrm{CW}$, Norton PA, et al. Risk factors for mesh/suture erosion following sacral colpopexy. AJOG. 2008;199(6):688. e1-. e5.
5. Lane FE. Repair of posthysterectomy vaginal vault prolapse. Obstet Gynecol.1962;20:72-6.

6. Visco AG, Weidner AC, Barber MD, Myers ER, Cundiff GW, Bump RC, et al. Vaginal mesh erosion after abdominal sacral colpopexy. AJOG.2001;184(3):297-302.

7. Miklos JR, Chinthakanan O, Moore RD, Mitchell GK, Favors S, Karp DR, et al. The IUGA/ICS classification of synthetic mesh complications in female pelvic floor reconstructive surgery: a multicenter study. Intl Urogyne colJ. 2016;27(6):933-8.

8. Timmons MC, Addison WA. Mesh erosion after abdominalsacral colpopexy. J Pelvic Surg. 1997;1:75-80. 